\author{
Military Technical College \\ Kobry El-Kobbah, \\ Cairo, Egypt.
}

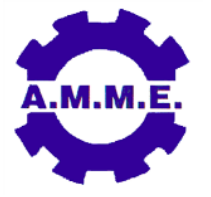
$16^{\text {th }}$ International Conference on Applied Mechanics and Mechanical Engineering.

\title{
2D VISCOUS SHAPE DESIGN OPTIMIZATION AND MESH ADAPTATION USING THE ADJOINT METHOD
}

\author{
K. Elsayed $^{\star} \dagger$, J. Miranda ${ }^{\ddagger}$, G. Ghorbaniasl ${ }^{\S}$ and C. Lacor ${ }^{\star \star}$
}

\begin{abstract}
The majority of the adjoint methods applications focus on research in aerodynamic shape design optimization such as flow in airfoil cascade. This paper presents the application of the adjoint method technique in simple test cases of engineering relevance. The goal of this work is to extend the adjoint method into more engineering applications for viscous and incompressible flow, and to further improve the performance. Two test cases of engineering relevance have been selected. (1) Mesh adaptation for 2-D lid-driven cavity. (2) Drag reduction using an optimized upstream rod. The adjoint method has been used to efficiently adapt the 2-D liddriven cavity mesh to minimize the discretization errors in calculating the swirl number. Based on the adaptation for the sensitivity magnitude, a uniform mesh of 1600 cells has been refined until a total 6586 cells, with finer mesh at the locations of highest sensitivities. The application of the adjoint method for the passive drag reduction problem results in a modified shape and position for the upstream cylinder. This change reduces the drag force on the main cylinder by $46 \%$.
\end{abstract}

\section{KEY WORDS}

Adjoint methods, Shape optimization, Mesh adaptation, Drag reduction.

Postdoc researcher, Department of Mechanical Engineering, Vrije Universiteit Brussel, Pleinlaan 2 -1050 Brussels- Belgium, Email: khairy.elsayed@vub.ac.be.

$\dagger$ Assistant Professor, Helwan University, Faculty of Engineering - Mattaria, Department of Mechanical Power Engineering, Masaken El-Helmia,11718 Cairo, Egypt, Email: kelsayed75@gmail.com.

‡ PhD student, Department of Mechanical Engineering, Vrije Universiteit Brussel, Pleinlaan 2 -1050 BrusselsBelgium, Email: joao.miranda@vub.ac.be.

$\S$ Professor, Department of Mechanical Engineering, Vrije Universiteit Brussel, Pleinlaan 2 -1050 BrusselsBelgium, Email: ghader.ghorbaniasl@vub.ac.be.

Professor, Department of Mechanical Engineering, Vrije Universiteit Brussel, Pleinlaan 2 -1050 BrusselsBelgium, Email: chris.lacor@vub.ac.be. 


\section{INTRODUCTION}

Due to the growth in computational power and numerical methods, aerodynamic shape optimization using numerical methods has become increasingly popular in engineering design [1]. Optimization methods can be classified into gradient (local) and non-gradient (global) methods depending on whether or not sensitivity analysis is applied [1].

A global optimization method may provide a globally optimal value within a specified design space. For example, Genetic Algorithm (GA) is widely accepted as a global optimization tool [2-4]. GA is generally computationally expensive when used to imitate an accurate evolutional process. For three dimensional aerodynamic design problems with many design variables, it requires a prohibitive amount of computational cost in evaluating experimental (or numerical) data at each design point. For this reason, a GA as an aerodynamic shape optimization tool is applicable to limited problems with a few design variables. A GA is usually implemented in conjunction with some meta-modeling technique such as RSM [5], Kriging [6,7] or other surrogate models [8]. Once a meta-model is constructed by a suitable mathematical function and experimental (or CFD) data points in the design space, it can predict new values without additional flow analysis. However, when a geometric shape is changed dramatically or there are many design variables, these modeling methods may entail a huge computational cost to obtain sufficient training data for building an accurate surrogate model. Furthermore, if the sample points (design of experiment) representing the objective function values are not proper, the design results could be poor.

The gradient-based optimization technique is quite popular in aerodynamic shape design mainly because the computational cost of an adjoint approach is essentially independent of the number of design variables [9-12]. This technique exhibits good convergence since it uses the gradient vector of the objective function, which provides the optimal direction in the design space. The method is particularly powerful in case of shape design (such as wing surface design), which usually involves many design variables. Jameson [9] pioneered a continuous adjoint approach, and applied it to aerodynamic shape optimization problems of several wing/body geometries with wing planform and surface design variables $[9,10]$. Mavriplis [13], Nielsen and Anderson [14] and Koc et al. [15] extended the discrete adjoint method to design problems of various complex geometries on unstructured mesh systems. In these applications, both continuous and discrete adjoint variable methods significantly improved the aerodynamic performance.

The majority of previous shape optimization studies focused on the external flow application while very limited studies applied the shape optimization (especially using the adjoint method approach) on the internal flow applications. Lee and Kim [12] applied the discrete adjoint method to obtain an optimal shape design for a subsonic S-shaped intake geometry. Zymaris et al. [16] used the S-shaped duct and the $90^{\circ}$ bend test cases to compare the calculated sensitivities using the widely used frozen turbulence adjoint (the variation in turbulent viscosity is assumed negligible) and their proposed turbulent adjoint using the Spalart - Allmaras turbulence model. Nevertheless, they did not present the optimal shapes for the tested cases. The present work focuses on the application of the gradient-based optimization technique 
and the adjoint method in the aerodynamic shape optimaization and mesh adaptation problems. The efficiency and capability of the adjoint method will be demonstrated through different test cases. As a starting point, a gentle introduction to the adjoint solver available in Fluent 14.5 will be given. Then, the adjoint method will be applied to many internal flow application of engineering relevance. Finally, in order to achieve accurate results, we will use the adjoint method for the mesh adaptation.

\section{ADJOINT METHOD}

\section{The Need of Adjoint Solver}

An adjoint solver is a specialized tool that extends the scope of the analysis provided by a conventional flow solver by providing detailed sensitivity data for the performance of a fluid system. In order to perform a simulation using a flow solvers, a user supplies the solver with the system geometry in the form of a computational mesh, specifies material properties and physics models, and configures boundary conditions of various types. The conventional flow solver, once converged, provides a detailed data set which describes the flow state governed by the flow physics that are being modeled [17].

The sensitivities of a fluid system provided by an adjoint solver satisfy a central need in gradient-based shape optimization. This makes an adjoint solver a unique and powerful engineering tool for design optimization. Adjoint data can also play a role in improving solver numerics. Regions of high sensitivity are indicative of areas in the flow where discritization errors can potentially have a strong effect. This information can be used to guide how best to refine a mesh to improve flow solution accuracy.

Once the adjoint is computed it can be used to guide intelligent design modifications to a system. After all, the adjoint sensitivity data provides a map across the entire surface of the geometry of the effect of moving the surface. Design modifications can be most effective if made in regions of high sensitivity since small changes will have a large effect upon the engineering quantity of interest. This principle of making changes to a system in proportion to the local sensitivity is the foundation for the simple gradient algorithm for design optimization.

\section{Discrete Adjoint Solver}

An adjoint method can be used to compute the derivative of an observation of interest for the fluid system with respect to all the user-specified parameters, with any changes that arise in the flow variables themselves eliminated. There are three key ingredients to consider when developing the method in the Fluent adjoint solver:

1. All of the user-specified inputs:

- All values set by a user in the boundary condition panels for each boundary in the problem.

- The computational mesh. More specifically the locations of the nodes of the mesh and how they define the edges, faces, and ultimately the cells used in the finite-volume computation. This includes both interior as well as boundary nodes.

- Material properties. 
- Model parameters such as model coefficients for turbulence models.

Note that the settings that define the problem are being distinguished from settings that define how the solution advancement is to be performed to converge the problem. Only the former are of interest here. For the sake of clarity, let us denote the vector of all of the values in the list by $\underline{c}$. These are considered to be the control variables for the problem, that is, the variables that a user can set explicitly that affect the solution. It is worth noting that the topological definition of the mesh is fixed-it is not considered to be a control variable here.

2. The governing equations for the fluid system: The main effort in a flow computation is in the determination of the flow state, namely the velocity, pressure, density and possibly other fluid-related variables. For a cell-centered finite-volume scheme, the flow state is defined at the cell centroids by a vector of real values. In the simplest case, these values are the pressure and flow velocity components. Let the vector of the variables in the $v$ th cell be denoted here by $q^{v}$ such as the static pressure and the velocity components. At convergence the flow variables satisfy Eq. (1).

$$
\mathbf{R}_{i}^{\mu}\left(\underline{q}^{0}, \underline{q}^{1} \cdots, \underline{q}^{M-1} ; \underline{c}\right)=0, \mu=0, \cdots, M-1, i=0, \cdots, L-1
$$

where $M$ is the number of cells in the problem, and there are $L$ conditions on each cell. This expression is a compact way of denoting conservation of mass and momentum and other constraints.

3. The engineering observation of interest (also called the objective function such as the pressure drop or drag force): Let

$$
\mathbf{J}\left(\underline{q}^{0}, \underline{q}^{1} \cdots, \underline{q}^{M-1} ; \underline{c}\right)
$$

denote a scalar of interest that depends both on the flow state $\underline{q}$ and perhaps directly on the control variables $\underline{c}$. It is assumed that the observable is differentiable with respect to both the flow and the controls.

The goal is to determine the sensitivity of the observation with respect to the user-specified control variables. What makes defining this relationship more challenging is the fact that changing the user inputs changes the flow, which indirectly changes the engineering observation. The adjoint method has a specific role in managing this chain of influences by providing a mechanism for eliminating the specific changes that happen in the flow whenever the inputs change.

If a variation $\delta c_{j}$ is introduced into the control variables then a linearization of the governing equations (Eq. (1)) shows that the variations in the flow state $\delta q_{j}^{v}$ must satisfy $\delta \mathbf{R}_{i}^{\mu}=0$ to satisfy the conservation equation, i.e., it satisfies Eq. (4).

$$
\begin{gathered}
\delta \mathbf{R}_{i}^{\mu}=0 \\
\frac{\partial \mathbf{R}_{i}^{\mu}}{\partial q_{j}^{\nu}} \delta q_{j}^{v}+\left.\frac{\partial \mathbf{R}_{i}^{\mu}}{\partial c_{j}}\right|_{q} \delta c_{j}=0
\end{gathered}
$$




$$
\frac{\partial \mathbf{R}_{i}^{\mu}}{\partial q_{j}^{\nu}} \delta q_{j}^{v}=-\left.\frac{\partial \mathbf{R}_{i}^{\mu}}{\partial c_{j}}\right|_{q} \delta c_{j}, \mu=0, \cdots, M-1, i=0, \cdots, L-1
$$

where there is an implied summation over $j, v$, and $I_{q}$ denotes that the flow solution is held constant while the derivative is taken. Meanwhile, if both the control variables and the flow state change, then the observation will change as follows.

$$
\delta \mathbf{J}=\frac{\partial \mathbf{J}}{\partial q_{j}^{v}} \delta q_{j}^{v}+\left.\frac{\partial \mathbf{J}}{\partial c_{j}}\right|_{q} \delta c_{j}
$$

The particular way in which the flow responds to the changes in the control variables can be computed using Eq. (4) only after specific changes, $\delta c_{j}$, have been chosen. It is prohibitive to consider solving Eq. (4) for more than a handful of prescribed changes $\delta c_{j}$ because of the excessive computing time that would be needed. However, when redesigning the shape of parts of a system there may be pressure to explore a large number of candidate modifications. This conflict is reconciled by eliminating the variations of the flow solution from the expression Eq. (5) and producing an explicit relationship between changes in the control variables and the observation of interest.

Introducing an arbitrary multiplier (the adjoint variable), $\tilde{q}_{i}^{\mu}$. Multiplying the adjoint variable by Eq. (3) and subtract the results from Eq. (5) will make no change in the calculated value of $\delta \mathbf{J}$ because the subtracted term equals zero.

$$
\delta \mathbf{J}=\frac{\partial \mathbf{J}}{\partial q_{j}^{v}} \delta q_{j}^{\nu}+\left.\frac{\partial \mathbf{J}}{\partial c_{j}}\right|_{q} \delta c_{j}-\left\{\left[\tilde{q}_{i}^{\mu} \frac{\partial \mathbf{R}_{i}^{\mu}}{\partial q_{j}^{\nu}}\right] \delta q_{j}^{\nu}+\left.\tilde{q}_{i}^{\mu} \frac{\partial \mathbf{R}_{i}^{\mu}}{\partial c_{j}}\right|_{q} \delta c_{j}\right\}
$$

Rearrange the terms in two groups of cause of change in $\mathbf{J}$ reads Eq. (7).

$$
\delta \mathbf{J}=\left(\frac{\partial \mathbf{J}}{\partial q_{j}^{\nu}}-\left[\tilde{q}_{i}^{\mu} \frac{\partial \mathbf{R}_{i}^{\mu}}{\partial q_{j}^{v}}\right]\right) \delta q_{j}^{v}+\left(\left.\frac{\partial \mathbf{J}}{\partial c_{j}}\right|_{q}-\left.\tilde{q}_{i}^{\mu} \frac{\partial \mathbf{R}_{i}^{\mu}}{\partial c_{j}}\right|_{q}\right) \delta c_{j}
$$

In order to eliminate the explicit dependance of $\delta \mathbf{J}$ on the change of the flow variables $\delta q_{j}^{\nu}$, we would like to make $\frac{\partial \mathbf{J}}{\partial q_{j}^{v}}-\left[\tilde{q}_{i}^{\mu} \frac{\partial \mathbf{R}_{i}^{\mu}}{\partial q_{j}^{\nu}}\right]$ equals zero. Equation (8) is the discrete adjoint equation. The variation of the objective function can be calculated as $\delta \mathbf{J}=\left(\left.\frac{\partial \mathbf{J}}{\partial c_{j}}\right|_{q}-\left.\tilde{q}_{i}^{\mu} \frac{\partial \mathbf{R}_{i}^{\mu}}{\partial c_{j}}\right|_{q}\right) \delta c_{j}$ which has no explicit dependance on the change in the flow variables.

$$
\left[\tilde{q}_{i}^{\mu} \frac{\partial \mathbf{R}_{i}^{\mu}}{\partial q_{j}^{\nu}}\right]=\frac{\partial \mathbf{J}}{\partial q_{j}^{\nu}}
$$

\section{Solution-Based Adaption Using The Adjoint Method}

An adjoint solution provides guidance on where best to adapt a computational mesh in order to accurately resolve quantities of engineering interest. 
Once the governing equations for the system (Eq. (1)) have been converged there remains a discritization error, $\varepsilon_{i}^{\mu}$, such that

$$
\mathbf{R}_{i}^{\mu}\left(\underline{q}^{0}, \underline{q}^{1} \cdots, \underline{q}^{M-1} ; \underline{c}\right)=\varepsilon_{i}^{\mu}, \mu=0, \cdots, M-1, i=0, \cdots, L-1
$$

While specific estimates for this discritization error may be tricky to define, it is often estimated to be $O\left(h^{p}\right)$, where $h$ is the local grid size, and $p$ is the order of the discritization scheme. That is, $p=1$ for a first-order scheme and $p=2$ for a second order scheme. Alternatively, $\varepsilon_{i}^{\mu}$ can be considered to be the residual associated with a solution that is not converged fully.

The correction to the flow field, $\delta q_{j}^{\nu}$, that compensates for this inhomogeneity is given by

from which it follows quickly that

$$
\frac{\partial \mathbf{R}_{i}^{\mu}}{\partial q_{j}^{\nu}} \delta q_{j}^{v}=-\varepsilon_{i}^{\mu}
$$

$$
\delta \mathbf{J}=\frac{\partial \mathbf{J}}{\partial q_{j}^{v}} \delta q_{j}^{v}=-\varepsilon_{i}^{\mu} \tilde{q}_{i}^{\mu}
$$

This simple expression provides an estimate of the effect of the presence of $\varepsilon_{i}^{\mu}$ on the observation $\mathbf{J}$.

The presence of discritization errors, or lack of convergence, on the engineering quantity of interest is assessed by weighting the inhomogeneous term by the local adjoint solution. It is clear that even in regions of the domain where the residuals or discritization errors are small, an accompanying adjoint velocity or pressure that is large in magnitude implies that there may be a significant source of error in the observable. A finer mesh in regions where the adjoint is large will reduce the influence of discritization errors that may adversely affect the engineering result of interest. In practice, adapting cells which have large magnitude adjoint velocity and/or adjoint pressure will achieve this goal.

\section{Shape Optimization Using The Adjoint Method}

Adjoint sensitivity data can be used to guide how to modify a system in order to improve the performance. The observable of interest can be made larger (maximization e.g. the lift force) or smaller (minimization e.g. the drag force or the pressure drop), depending upon the engineering goal.

A common strategy for deciding how to modify the system is based on the gradient algorithm. The underlying principle is quite simply that modifying a system in a manner to which it is most sensitive maximizes the effect of the change. The change to a control variable is made in proportion to the sensitivity of the value of interest with respect to that control variable.

Denote the sensitivity of the cost with respect to shape by: 


$$
\delta \mathbf{J}=\frac{\partial \mathbf{J}}{\partial x_{j}^{n}} \delta x_{j}^{n}
$$

where $x_{j}^{n}$ is the $j$ th coordinate of the $n$th node in the mesh. Here $x_{j}^{n}$ is a notation for the subset of the control variables $c_{j}$ for the system that correspond to mesh node positions. Then an adjustment

$$
\delta x_{j}^{n}=\lambda \frac{\partial \mathbf{J}}{\partial x_{j}^{n}}
$$

will provide the maximum adjustment to $\mathbf{J}$ for given $L^{2}$ norm of $\partial x_{j}^{n}$, where $\lambda$ is an arbitrary scaling factor. Note that $\lambda$ can be picked to be positive or negative depending upon whether $\mathbf{J}$ is to be increased or decreased respectively. This is essentially a statement of the method of steepest descent.

Furthermore, the change is estimated to first order to be (from Eq. (13) into Eq. (12))

$$
\delta \mathbf{J}=\lambda \frac{\partial \mathbf{J}}{\partial x_{j}^{n}} \frac{\partial \mathbf{J}}{\partial x_{j}^{n}}
$$

For a sufficiently small adjustment, the change to the observation will strictly have the same sign as the scaling factor $\lambda$, provided the gradient is not identically zero.

It is noted that in practical cases, the field $\frac{\partial \mathbf{J}}{\partial x_{j}^{n}}$ can be noisy. If the noisy field is used directly to modify a boundary shape using (Eq. (13)), then the modified surface can have many inflections. This is not helpful for engineering design work. In this case, the use of mesh morphing technology is a must not only to smooth the sensitivity field, but also to provide smooth boundary and interior mesh deformation.

\section{Smoothing and Mesh Morphing}

For typical engineering problems, the shape sensitivity field can have smoothness properties that are not adequate to define a shape modification. Mesh morphing technology is used in a two-fold role. The first role is as a smoother for the surface sensitivity field. The second role is to provide smooth distortions not only of the boundary mesh, but also the interior mesh. This approach is very appealing since it functions for arbitrary mesh cell types.

A rectangular control volume is picked that encloses the boundary, or part of the boundary, whose shape is to be modified. A regular array of control points is then distributed in the control volume.

The properties of Bernstein polynomials are then invoked to define a local coordinate system mapping. The standard coordinates, $\underline{x}^{k}$, of each boundary and interior node in the mesh, lying within the control volume, are defined by a local coordinate, $\left(u^{k}, v^{k}\right)$. In a control volume, with $l \times m$ control points, the linear relationship between the $k$ th grid node position and the $i j$ th control point location is:

$$
\underline{x}^{k}=\sum_{i, j=0}^{\ell, m} \underline{\xi}^{i j} B_{i, \ell}\left(u_{k}\right) B_{j, m}\left(v_{k}\right)
$$


where $B_{i, l}(u)$ is the th Bernstein polynomial of degree $l$,

$$
B_{i, \ell}(u)=\left(\begin{array}{l}
\ell \\
i
\end{array}\right) u^{i}(1-u)^{n-i}
$$

and $\underline{\xi}^{i j}$ denotes the coordinate of the $i j$ th control point.

If the regular array of control points is modified by moving one or more control points, the mapping provides a smooth repositioning of the grid nodes.

It is has already been discussed before how the variation in the observation varies with mesh node locations:

$$
\delta \mathbf{J}=\frac{\partial \mathbf{J}}{\partial x_{j}^{n}} \delta x_{j}^{n}
$$

Using the mapping between the mesh nodes and control points, shows that

where

$$
\delta \mathbf{J}=\sum_{i, j=0}^{\ell, m} \delta \underline{\xi}^{i j} \cdot \underline{\widetilde{W}}^{i j}
$$

$$
\underline{\widetilde{W}}^{i j}=\sum_{n} \frac{\partial \mathbf{J}}{\partial \underline{x}^{n}} B_{i, \ell}\left(u_{n}\right) B_{j, m}\left(v_{n}\right)
$$

is the control point sensitivity field and $\delta \xi^{i j}$ denotes the adjustment to the control point position. Since there are typically fewer control points than grid nodes, the summation operation in Eq. (19) has a smoothing effect on the sensitivity field.

A simple gradient algorithm based on choosing

$$
\delta \underline{\xi}^{i j}=\lambda \underline{\widetilde{W}}^{i j}
$$

leads to a smooth boundary mesh deformation that improves the design, for $\lambda$ sufficiently small. The added benefit is that a smooth volume mesh deformation is also defined.

Continuity of the mesh displacement derivatives at the control volume perimeter to order $p$ can be preserved by explicitly setting the control point displacement field to be zero for control points in layers adjacent to the control volume boundary. If no control point displacement is permitted in layers 0 through $p$ and $\ell-p$ through $\ell$ in the $x$-coordinate direction, and likewise in the $y$-direction, the properties of Bernstein polynomials guarantee the enforcement of the continuity condition.

\section{The Adjoint Solver Procedure}

The main steps for shape modification process using the adjoint solver:

- Start with a basic geometry and solve the flow equations until conversion.

- Select the objective function (observables) and run the adjoint solver to estimate the sensitivities.

- Postprocess the adjoint solution data to study qualitatively and quantitatively the effect of many types of change that may be imposed on a system.

- Modify the geometry by selecting the zone to be modified, the boundary continuity 
condition (default $=2$ ), the scale factor (default $=1$ ) and the bounding box for the region of mesh deformation.

- Repeat the steps until no considerable change in the expected change in the observable.

In case of solution-based adaptation, after calculate the sensitivities, the mesh can be adapted based on the sensitivities for the region of high sensitivity. Before start applying the adjoint method to the selected test cases, it is worth to define some widely used variables in the context of sensitivities [17].

Shape Sensitivity Magnitude: is the magnitude of the sensitivity of the observable with respect to a deformation applied to the mesh (both boundary and interior mesh). When plotted on the surface of a body, the locations where this quantity is large indicates where small changes to the surface shape can have a large effect on the observable of interest. It is often observed that the magnitude varies by many orders of magnitude.

Log10 (Shape Sensitivity Magnitude): In view of the large range of values possible for the shape sensitivity magnitude a convenience function which plots $\log _{10}$ of the magnitude is provided. This allows the importance of the surfaces in a domain to be ranked more easily based on how they affect the observation of interest when they are reshaped.

Sensitivity to Flow Blockage: This function is provided as a convenient tool for identifying portions of the flow domain where the introduction of blockages or obstructions in the flow can affect the observation of interest. Consider a blockage in the flow that generates a reaction force on the flow that is proportional to the local flow speed, and acting in the opposite direction to the local flow: $F=-\alpha(r) v$ where $\alpha$ is a local coefficient for the reaction force. The local contribution of this force on the observation of interest is determined by the vector product of this force with the adjoint velocity field. The flow blockage field that is plotted is $-v \cdot \tilde{v}$, namely the negative of the vector product of the flow velocity and the adjoint velocity (Cell Value) [17].

For more details about the Fluent adjoint solver, we refer to the Fluent manual [17].

\section{RESULTS AND DISCUSSION}

\section{Mesh Adaptation For 2-D Lid-Driven Cavity}

The lid driven cavity flow (Fig. 1(a)) is one of the most studied fluid problems in computational fluid dynamics field since the early work of Burggraf [18]. The simplicity of the geometry of the cavity flow makes the problem easy to code and apply boundary conditions. Even though the problem looks simple in many ways, the flow in a cavity retains all the flow physics with counter rotating vortices appear at the corners of the cavity. Driven cavity flow serve as a benchmark problem for numerical methods in terms of accuracy of the numerical results [19]. This flow configuration is also relevant to a number of industrial applications [20-22]. In the literature it is possible to find numerous studies on the driven cavity flow [23].

In this study, the adjoint solver was used to adapt a uniform (coarse) mesh in order to accurately predict the flow feature. Whereas it is well known a fine mesh close to the 
corners are needed to accurately capture the flow features in the corners, we started with a uniform mesh consists of 1600 quadrilateral cells as shown in Fig. 1(b). In the adjoint solver, we selected the swirl as the observable (objective function), from the available list of observables in the Fluent-Adjoint solver 14.5.7. The swirl is defined as the moment of the mass flow relative to an axis defined by a point, $r_{c}$, and a direction $d$ i.e., $S=\int \rho(\vec{r} \times \vec{u}) \cdot d \overrightarrow{\mathrm{d}} V$ where $V$ denotes the volume over which the integration is made, and $r$ denotes the relative position to the point $r_{c}$ [17]. In this study, $V$ is the flow domain and $r_{c}=(0,0)$ i.e., the center point.

The stream function, vorticity contours and the Log10 for the shape sensitivity magnitude for the original mesh are shown in Fig. 1 for a laminar flow with Reynolds number of 1000, where all walls are fixed with no slip conditions and the upper boundary moves to the right at a fixed velocity of $1 \mathrm{~m} / \mathrm{s}$.

For accurate calculation of the swirl (around point $(0,0)$ ), the region of high gradient in the swirl was adapted. The mesh cells of high contribution to the change in the swirl was marked for adaptation. The adaptation performed using the iso-value adaption option available in Fluent for the Log10 of the shape sensitivity magnitude variable from 0.0 to 1.964 which is the maximum value in the domain for the Log10 of the shape sensitivity magnitude. This adaptation refined 352 cells (cf. Fig. 1(f)). In the second cycle of adaptation, 964 cells were marked for refinement (using the iso values from 0.0 to 2.463). In total, the original mesh of 1600 cells ( $S=-0.0819$ $\mathrm{kg}-\mathrm{m}^{2} / \mathrm{s}$ ) became a locally refined mesh of 6586 cells $\left(S=-0.0895 \mathrm{~kg}-\mathrm{m}^{2} / \mathrm{s}\right)$. The adapted mesh are shown in Fig. 2(a) which is very close to that obtained by Magalhaes et al. [25] (cf. Fig. 7(a) in [25] with 6910 cells). Magalhaes et al. [25] employed a posteriori error estimation criterion based on the assessment of the goodness-of-fit of the least squares regression in order to perform the variables profile reconstruction and to be capable of detecting both large-scale and small-scale flow phenomena in the cavity problem.

The obtained stream function, vorticity contours and the Log10 of the shape sensitivity magnitude are shown in Figs. 2 (b,c and d) respectively. The velocity magnitude at the horizontal and vertical centerline for the two meshes have been plotted against the CFD simulation results of Ghia et al. [24] who used 16641 cells. The results shows the superior performance of the used technique.

\section{Drag Reduction Using Passive Control Technique}

Most structures on land and in the ocean are confronted by a fluid flow. Vibrations of these structures due to fluid flows reduce the life of the respective installations and must therefore be taken into account in the design of the structure. An elementary shape of a structure (or a component of a structure) is a circular cross-section. The tandem arrangement of two circular cylinders is a basic example of an array of multiple structures. The common use of the cylindrical-shaped body in various fields of engineering stimulated the investigation on flow around two circular cylinders in a tandem arrangement. Within these studies, considerable efforts have been devoted to improving aerodynamic characteristics such as drag and lift forces by controlling the flow around the cylinder.

In general, flow control techniques for reducing the aerodynamic drag exerted on a 
bluff body are classified into two types, referred to as active and passive control. Active control methods control the flow by supplying external energy through means such as jet blowing. Passive control methods control the flow by modifying the shape of the body or by attaching additive devices such as a control rod or roughness elements onto the body [27]. Active control requires complex mechanical devices that supply external power to the flow. Therefore, compared with the active flow control method, passive control is simpler and easier to implement.

Previous studies have clearly shown that inclusion of a control rod upstream of the main body is a very simple and effective method for controlling the flow around a bluff body such as a circular cylinder [28-38]. According to the best of the authors knowledge, there is no optimization studies for this particular problem have been performed before especially using the adjoint methods.

Aly and Elsayed [38] investigated experimentally and simulated numerically the aerodynamic characteristics of two cylinders in a tandem arrangement were subjected to a uniform flow at a Reynolds number of $5.7 \times 10^{4}$. This Reynolds number is within the range in which fluid forces acting on a single cylinder are comparatively insensitive to the change in the Reynolds number. Five diameters of control rods namely; 4, 5, 6, 7 and $10 \mathrm{~mm}$ have been investigated. Wake velocity and surface pressure distributions were measured with gap width distance ranged from 0 to 80 $\mathrm{mm}$. From this study, we have selected one test case to be used as a baseline for the shape optimization using the adjoint method. The rod diameter equals $5 \mathrm{~mm}$ and a gap of $20 \mathrm{~mm}$ whereas the cylinder diameter equals 1 inch $(D=25.4 \mathrm{~mm})$. The ratio of the rod to cylinder diameter $\frac{d}{D}=\frac{5}{25.4}=0.197$ and the gap distance to the cylinder radius $\frac{l}{D}=\frac{20}{25.4}=0.79$ and the corresponding center to center distance $\frac{L}{D}=\frac{35.2}{25.4}=$ 1.39. The geometrical arrangement of the two cylinders and the coordinate system used in this study are shown in Fig. 3.

\section{Numerical settings}

The domain extends from $10 \mathrm{D}$ at the left to $30 \mathrm{D}$ at the right. The symmetry boundary conditions are located at $\pm 16 D$ from the cylinder centerline. The inlet velocity is $32 \mathrm{~m} / \mathrm{s}$ with $2 \%$ turbulent intensity and a hydraulic diameter equals the cylinder diameter for the left boundary (velocity inlet). The boundary condition for the right boundary is pressure outlet whereas the top and lower surfaces are symmetry (zero shear stress). The air density equals $1.25 \mathrm{~kg} / \mathrm{m}^{3}$ and viscosity of $1.827 \times 10^{-5}$ $\mathrm{kg} / \mathrm{ms}$. The unsteady simulation performed using a time step, $\Delta t=10^{-5}$. The mesh contains 114511 unstructured triangular grid cells as shown in Fig. 4. Table 1 summarize the solution methods used in this simulation.

The unsteady flow has been simulated using $k-\omega$ SST model [39] for the unsteady incompressible flow. A perturbation (a velocity of opposite direction on the upper and lower boundaries are imposed at $t=0$ ) has been used to initiate the Karman vortex street.

Figure 5 shows the converged flow results for the instantaneous static pressure, turbulent kinetic energy and the stream function. Where the Karman vortex street is clearly shown. The time averaged mean flow pattern is also given in Fig. 5. 
Figure 5(b) shows no vortex street exist in front of the cylinder (in the wake of the control rod). This pattern of flow is known as the cavity mode [40]. The existence of this cavity flow highly reduce the drag force on the main cylinder with respect to the isolated cylinder (without control rod).

Due to the limitation of the Fluent-adjoint solver, throughout the shape optimization process, the steady incompressible turbulent flow has simulated using the standard $\mathrm{k}-\varepsilon$ model with standard wall function.

For the adjoint settings: the boundary continuity is 2 . The bounding box for the mesh adaptation is from -0.0549 to 0.0549 in $x$ and $y$ directions respectively with 10 points in each direction as shown in Fig. 6(a). The scale factor equals 5 . The final shape has been obtained after 10 cycles of calculate the flow after shape modification and calculate the shape sensitivities using the adjoint solver. For both the governing and adjoint equation each cycle stops when the maximum residual falls below $10^{-5}$. The optimal shape and location of the rod for minimum drag on the cylinder is given in Fig. 6.

Figure 6 shows the flow pattern for the optimal geometry as well as the time-averaged static pressure coefficient for the cylinder and the rod. The cylinder-rod gap for the baseline and the optimal geometry gives rise to the cavity mode as is clear from the contour plot of streamline (Fig. 6(b)).

The location and shape of the modified rod as shown in Fig. 6(c). In the optimal geometry not only the rod cross section becomes oval instead of circular but also located closer to the cylinder. Moreover, its major axis is tilted down. This new geometry causes a break of the cavity mode by allowing a narrow stream of fluid by passing the rod and flowing directly across the cylinder. This will increase the flow kinetic energy in the boundary layer and consequently retard the separation point.

The Log10 shape sensitivity magnitude for both the baseline and the optimum geometry are shown in Fig. 6(d), where the modified geometry depicts lower values for the Log10 shape sensitivity magnitude. This means that any further shape modification will results in less change in the drag force.

Figure 7 shows the time-average pressure coefficient $C_{p}=\frac{p_{\theta}-p_{\infty}}{\frac{1}{2} \rho V_{\infty}^{2}}$ and th skin friction coefficient $C_{f}=\frac{\tau_{w}}{\frac{1}{2} \rho V_{\infty}^{2}}$ where $p_{\theta}$ is the time average static pressure at angle $\theta$ from the upstream flow, $V_{\infty}, p_{\infty}$ is the free stream static pressure and velocity respectively. $\tau_{w}$ is the wall shear stress and $\rho$ is the air density. The following remarks can be drawn from Fig. 7:

- The flow on the upper and lowered sides of the rod is symmetric (both $C_{p}$ and $C_{f}$ ). Whereas the flow around the cylinder is asymmetric.

- The stagnation point for the rod is located at $\theta=0$. The reattachment points (stagnation points on the cylinder) are $\theta=12.5$ and 65 for the baseline, whereas $\theta=22.5$ for the optimal geometry. The separation angle $\theta=155$ for both cylinders (baseline and optimal). The location of minimum pressure coefficient, $\left.\theta\right|_{c_{p_{\min =-175}}}=120$ and 325 for the cylinder in the baseline geometry and $\left.\theta\right|_{c_{p_{\text {min }=-1.25}}}=115$ and 320 for the cylinder in the optimal geometry. 
- The cylinder base pressure coefficient $\left.C_{p}\right|_{\theta=180}=-1.1$ for the baseline geometry and increases to $\left.C_{p}\right|_{\theta=180}=-0.5$. This increase in the base pressure reduces the pressure drag on the cylinder.

Table 2 presents the drag forces before and after optimization. Modifying the shape and orientation of the rod reduce the drag force on the cylinder by $46 \%$ and $28 \%$ on the rod due to the change in the pressure (form) drag.

Due to fluid structure interaction, the separated flow around the cylinders causes structure oscillation which is clear from the variation of the forces on the bluff bodies. The variation of the lift coefficient $\left(C_{L}=\frac{F_{L}}{\frac{1}{2} \rho U^{2} D}\right.$ where $F_{L}$ denotes the lift force, $U$ is the free inlet velocity, $\rho$ is the air density and $D$ is the cyclone diameter) is shown in Fig. 8 for both the baseline and optimal geometry. Figure 8 presents the power spectral density of the Strouhal number $S_{t}=\frac{f D}{U}$ where $f$ is the frequency of vortex shedding which can be calculated from the oscillation frequency of the lift force [41].

It is worth to mention that the the rod shape optimization not just reduced the drag force but also the oscillations of the cylinder. The Strouhal number value $S_{t}$ on the cylinder reduced from 0.244 for the baseline geometry to 0.223 for the optimal geometry as shown in Fig. 8.

\section{CONCLUSION}

In the present paper, we have presented an efficient, computational fluid dynamics (CFD)-based shape optimization method using the adjoint solver available in the Fluent solver. The following conclusions have been drawn from the selected test cases:

Mesh adaptation for 2-D lid-driven cavity: The adjoint method has been used to efficiently adapt the mesh to minimize the discritization errors in calculating the swirl number. Based on the adaptation for the sensitivity magnitude, a uniform mesh of 1600 cells has been adapted to be 6586 cells with finer mesh at the location of highest sensitivities.

Drag reduction using upstream rod: The drag force on the cylinder has been reduced by modifying the shape of the upstream rod by $46 \%$. Moreover, the Strouhal number on the cylinder reduced from 0.244 for the baseline to 0.223 .

\section{ACKNOWLEDGMENT}

This research work was partially funded by the Flemish Science Foundation (FWO) under the grant GOB71.13N. This support is gratefully acknowledged. 


\section{REFERENCES}

[1] J. Yim, B. J. Lee, C. Kim, Exploring multi-stage shape optimization strategy of multi-body geometries using kriging-based model and adjoint method, Computers \& Fluids 68 (0) (2012) 71-87.

[2] J. H. Holland, Adaptation in Natural and Artificial Systems, The University of Michigan Press, Ann Arbor, 1975.

[3] H.-S. Chung, J. J. Alonso, Multiobjective optimization using approximation model-based genetic algorithms, in: AIAA 2004-4325, 10th AIAA/ISSMO Symposium on Multidisciplinary Analysis and Optimization, 2004.

[4] J. McCall, Genetic algorithms for modelling and optimisation, Journal of Computational and Applied Mathematics 184 (1) (2005) 205-222.

[5] R. H. Myers, D. C. Montgomery, C. M. Anderson-Cook, Response Surface Methodology: Process and Product Optimization Using Designed Experiments, 3rd Edition, Wiley series in probability and statistics, Wiley, Hoboken, N.J., 2009.

[6] J. Sacks, W. J. Welch, T. J. Mitchell, H. P. Wynn, Design and analysis of computer experiments, Statistical science 4 (4) (1989) 409-435.

[7] K. Elsayed, C. Lacor, Robust parameter design optimization using Kriging, RBF and RBFNN, in: Eleventh International Conference of Fluid Dynamics (ICFD11), Alexandria, Egypt, 2013.

[8] K. Elsayed, C. Lacor, CFD modeling and multi-objective optimization of cyclone geometry using desirability function, artificial neural networks and genetic algorithms, Applied Mathematical Modelling 37 (8) (2013) 5680-5704, http://dx.doi.org/10.1016/j.apm.2012.11.010.

[9] A. Jameson, Aerodynamic design via control theory, Journal of Scientific Computing 3 (1988) 233-260.

[10] K. Leoviriyakit, A. Jameson, Aerodynamic shape optimization of wings including planform variations, AIAA paper 210.

[11] W. K. Anderson, V. Venkatakrishnan, Aerodynamic design optimization on unstructured grids with a continuous adjoint formulation, Computers \& Fluids 28 (4) (1999) 443-480.

[12] B. J. Lee, C. Kim, Automated design methodology of turbulent internal flow using discrete adjoint formulation, Aerospace Science and Technology 11 (2) (2007) 163-173.

[13] D. J. Mavriplis, Discrete adjoint-based approach for optimization problems on three-dimensional unstructured meshes, AIAA journal 45 (4) (2007) 741-750.

[14] E. J. Nielsen, W. K. Anderson, Recent improvements in aerodynamic design optimization on unstructured meshes, AIAA journal 40 (6) (2002) 1155-1163.

[15] S. Koc, H.-J. Kim, K. Nakahashi, Aerodynamic design optimization of wing-body configurations, AIAA paper 331.

[16] A. Zymaris, D. Papadimitriou, K. Giannakoglou, C. Othmer, Continuous adjoint approach to the spalart-allmaras turbulence model for incompressible flows, Computers \& Fluids 38 (8) (2009) $1528-1538$.

[17] ANSYS, ANSYS FLUENT Adjoint Solver, ANSYS, Inc., Southpointe, 275 Technology Drive, Canonsburg, PA 15317, USA (2013).

[18] O. R. Burggraf, Analytical and numerical studies of the structure of steady separated flows, J. Fluid Mech 24 (1) (1966) 113-151.

[19] O. Botella, R. Peyret, Benchmark spectral results on the lid-driven cavity flow, Computers \& Fluids 27 (4) (1998) 421-433.

[20] N. Alleborn, H. Raszillier, F. Durst, Lid-driven cavity with heat and mass transport, International journal of heat and mass transfer 42 (5) (1999) 833--853.

[21] P. Zdanski, M. Ortega, N. G. Fico Jr, Numerical study of the flow over shallow cavities, Computers \& fluids 32 (7) (2003) 953-974.

[22] M. Cheng, K. Hung, Vortex structure of steady flow in a rectangular cavity, Computers \& Fluids 35 (10) (2006) 1046-1062. 
[23] E. Erturk, Discussions on driven cavity flow, International journal for numerical methods in fluids 60 (3) (2009) 275-294.

[24] U. Ghia, K. N. Ghia, C. Shin, High-re solutions for incompressible flow using the navier-stokes equations and a multigrid method, Journal of computational physics 48 (3) (1982) 387-411.

[25] J. P. Magalhaes, D. Albuquerque, J. Pereira, J. C. Pereira, Adaptive mesh finite-volume calculation of $2 \mathrm{~d}$ lid-cavity corner vortices, Journal of Computational Physics 243 (2013) 365-381.

[26] K. Elsayed, C. Lacor, Numerical modeling of the flow field and performance in cyclones of different cone-tip diameters, Computers \& Fluids 51 (1) (2011) 48--59.

[27] H. Sakamoto, H. Haniu, K. Tan, An optimum suppression of fluid forces by controlling a shear layer separated from a square prism, ASME Transactions Journal of Fluids Engineering 113 (1991) 183-189.

[28] T. Igarashi, T. Tsutsui, Flow control around a circular cylinder by a new method (2nd report, fluids forces acting on the cylinder), Trans. JSME 55 (511) (1989) 708-714.

[29] T. Igarashi, T. Tsutsui, Flow control around a circular cylinder by a new method (3rd report, properties of the reattachment jet), Trans. JSME 57 (533) (1989) 8-13.

[30] H. B. Lee, S. J. Lee, Flow structure of modified cylinder wake by a small control cylinder, in: Asian Congress of Fluid Mechanics, 6 th, Singapore, 1995, pp. 1608-1611.

[31] M. Zdravkovich, Review of flow interference between two circular cylinders in various arrangemets, ASME Transactions Journal of Fluids Engineering 99 (1977) 618-633.

[32] T. Igarashi, Drag reduction of a square prism by flow control using a small rod, Journal of wind engineering and industrial aerodynamics 69 (1997) 141-153.

[33] T. Tsutaui, T. Igarashi, Drag reduction of a circular cylinder. 2nd report. effect of reynolds number., Trans. JSME 61 (586) (1995) 2069-2075.

[34] T. Tsutaui, T. Igarashi, Enhancement of heat transfer and reduction of drag of a circular cylinder (flow control using a small rod), Trans. JSME 62 (597) (1996) 1802-1809.

[35] A. Prasad, C. Williamson, A method for the reduction of bluff body drag, Journal of wind engineering and industrial aerodynamics 69 (1997) 155-167.

[36] S. Mittal, V. Kumar, A. Raghuvanshi, Unsteady incompressible flows past two cylinders in tandem and staggered arrangements, International Journal for Numerical Methods in Fluids 25 (11) (1997) 1315-1344.

[37] H. Sakamoto, H. Haniu, Optimum suppression of fluid forces acting on a circular cylinder, Journal of fluids engineering 116 (2) (1994) 221-227.

[38] M. H. Aly, K. Elsayed, Experimental and numerical investigation of drag reduction on a circular cylinder, Engineering Bulletin, Faculty of Engineering, Mattaria, Helwan University, Egypt.

[39] F. R. Menter, Two equation eddy-viscosity turbulence models for engineering applications, AIAA Journal 32 (8) (1994) 1598-1605.

[40] S.-J. Lee, S.-I. Lee, C.-W. Park, Reducing the drag on a circular cylinder by upstream installation of a small control rod, Fluid Dynamics Research 34 (4) (2004) 233 -250.

[41] B. G. Dehkordi, H. S. Moghaddam, H. H. Jafari, Numerical simulation of flow over two circular cylinders in tandem arrangement, Journal of Hydrodynamics, Ser. B 23 (1) (2011) $114-126$. 
Table 1. The used solution methods for drag reduction in Fluent 14.5.

\begin{tabular}{lcccccc}
\hline For & Pressure velocity coupling & \multicolumn{3}{c}{ Spatial discretization } \\
\cline { 3 - 6 } & SIMPLE & Gradient & Pressure & Momentum & Turbulent kinetic energy & Turbulent dissipation rate \\
\hline Scheme & & Green-Gauss Node based & Standard & QUICK & First order upwind & First order upwind \\
\hline
\end{tabular}

Table 2. The drag force for the rod and cylinder $[\mathrm{N}]^{a}$.

\begin{tabular}{lcccccc}
\hline & \multicolumn{3}{c}{ Cylinder } & & \multicolumn{3}{c}{ Rod } \\
\cline { 2 - 6 } & Pressure & Viscous & Total & & Viscous & Total \\
\cline { 2 - 6 } Baseline & $14.578(1.12)$ & 0.457 & $15.035(1.156)$ & 1.602 & 0.067 & 1.669 \\
Optimal & 7.603 & 0.455 & $8.058(0.619)$ & 1.091 & 0.106 & 1.197 \\
\hline \% Reduction & 47.846 & 0.437 & 46.405 & 31.897 & -58.208 & $\mathbf{2 8 . 2 8 0}$ \\
\hline
\end{tabular}

${ }^{a}$ The value in parentheses is the drag coefficint $C_{D}$.

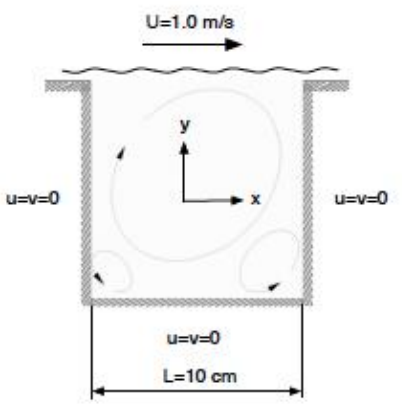

(a) 2D Lid driven cavity flow

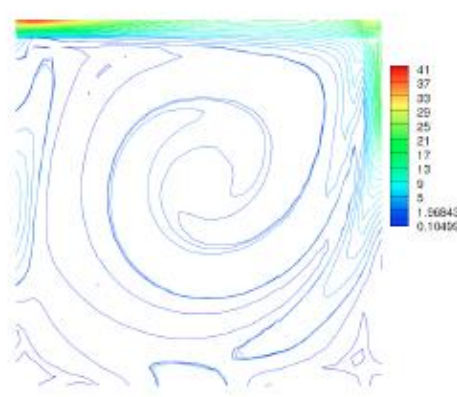

(d) Vorticity magnitude for the original mesh

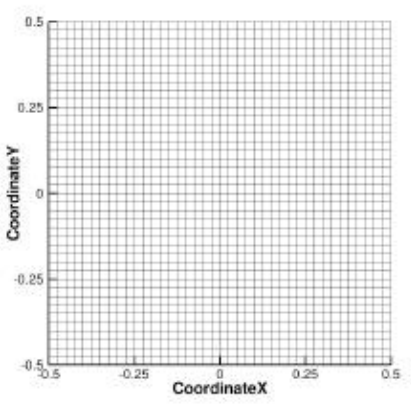

(b) Original mesh (1600 cells)

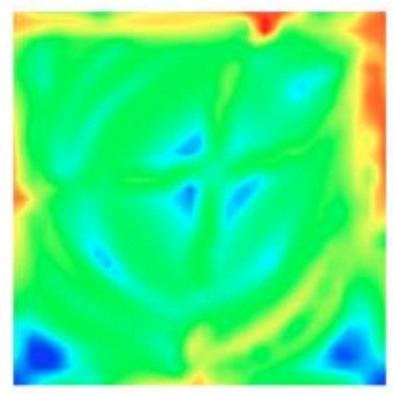

(e) Log10 for the shape sensitivity magnitude for the original mesh

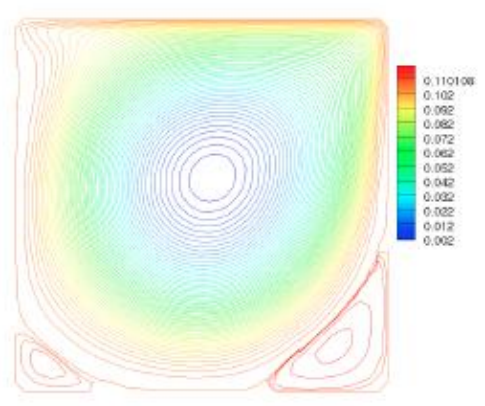

(c) Stream function for the original mesh

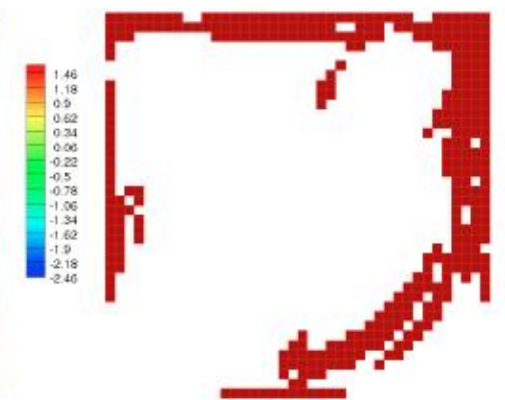

(f) 352 Marked cells for adaptation

Fig. 1. 2D lid driven cavity. 


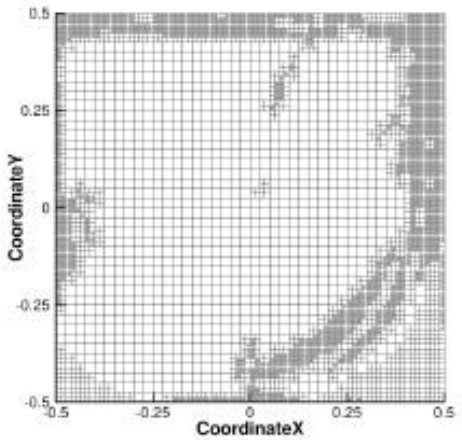

(a) Adapted mesh (6586 cells)

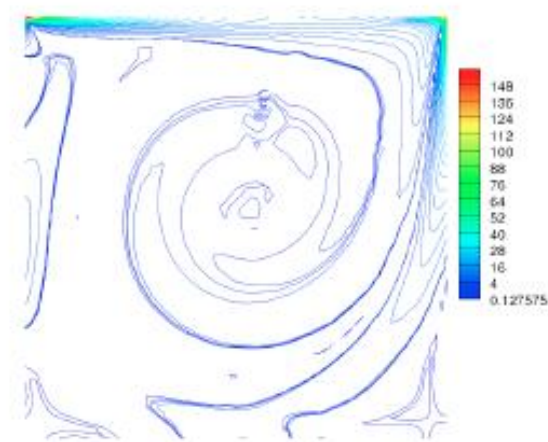

(c) Vorticity magnitude for the adapted mesh

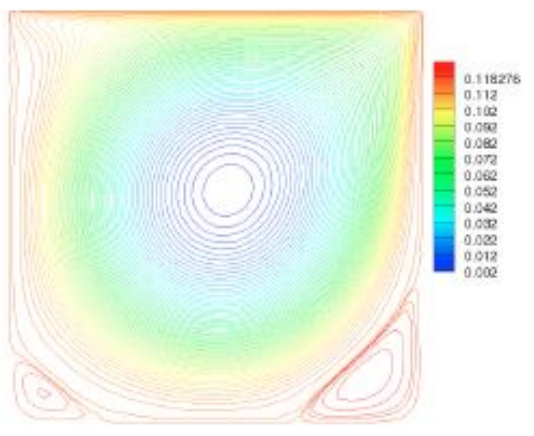

(b) Stream function for the adapted mesh

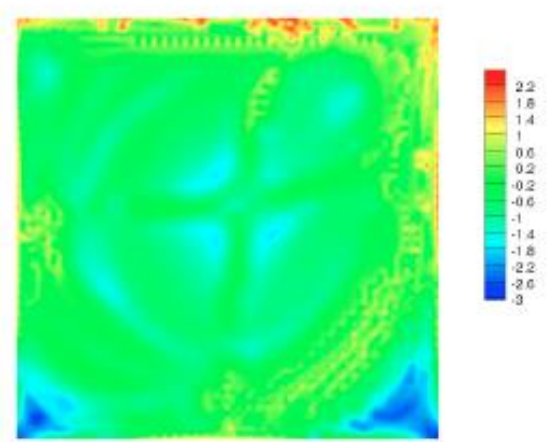

(d) Log10 for the shape sensitivity magnitude for the adapted mesh

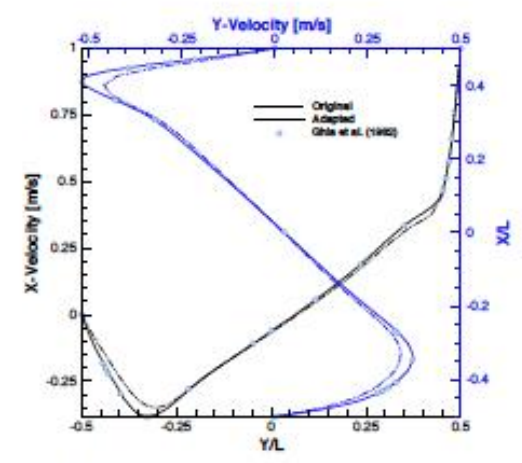

(e) The velocity magnitude at the horizontal and vertical centerline for the two meshes in addition to Ghia et al. [24] results using 16641 cells

Fig. 2. The results on the adapted mesh for the $2 \mathrm{D}$ lid-driven cavity problem.
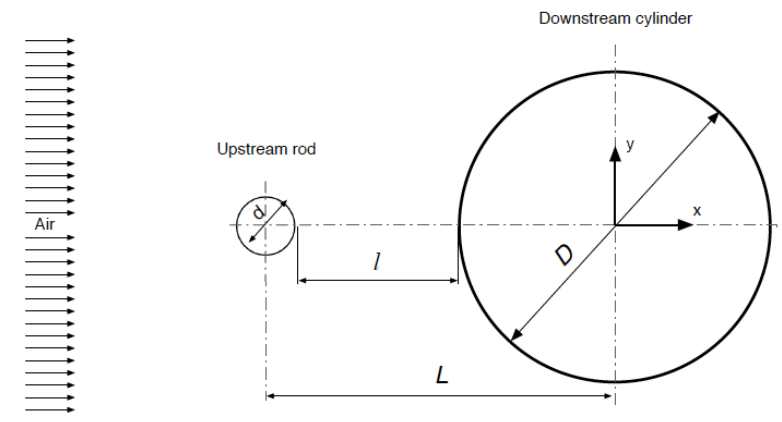

Fig. 3. Upstream control rod and downstream main cylinder, and definitions of symbols. 


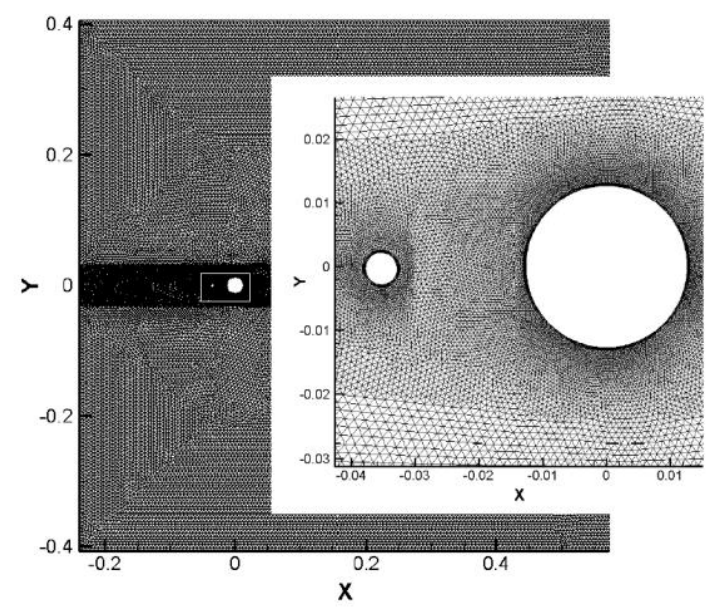

Fig. 4. The mesh for the original geometry and zoom around the cylinders.

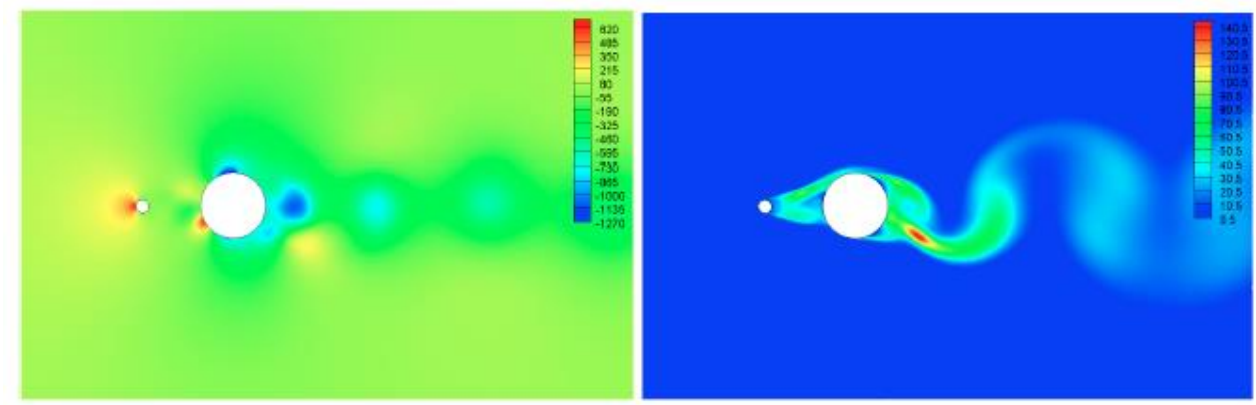

(a) Contour plot for the instantaneous static pressure (left) and turbulent kinetic energy (right)

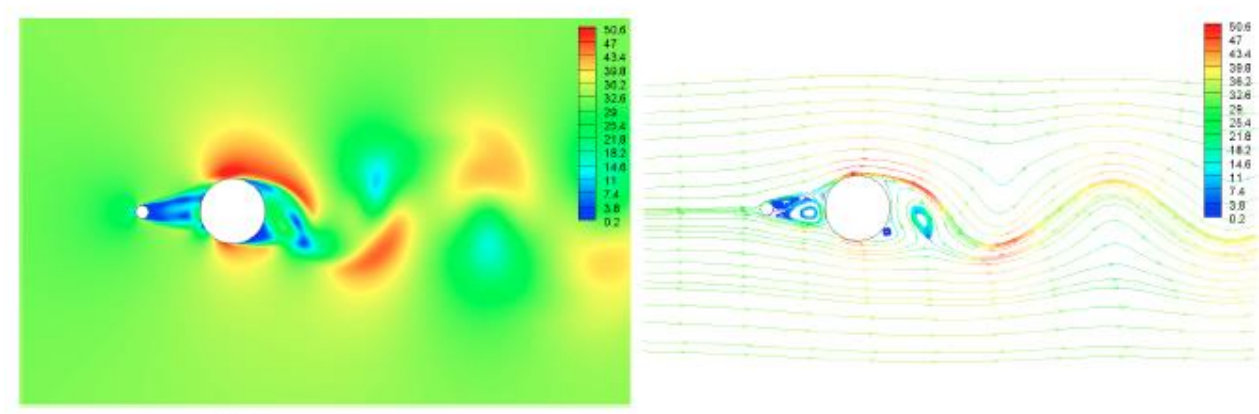

(b) Contour plot and stream function plot for the instantaneous velocity magnitude

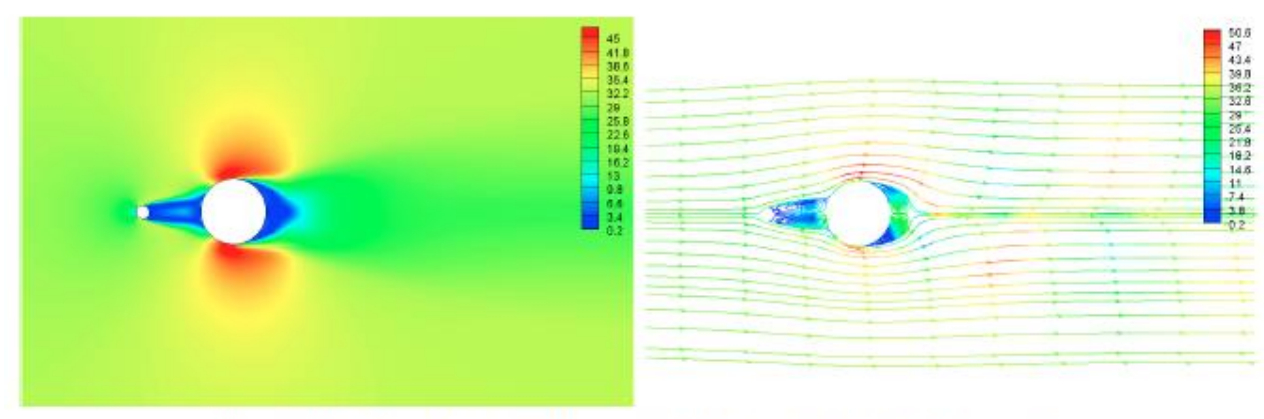

(c) Contour plot and stream function plot for the time-averaged velocity magnitude

Fig. 5. The flow pattern using the baseline geometry. 

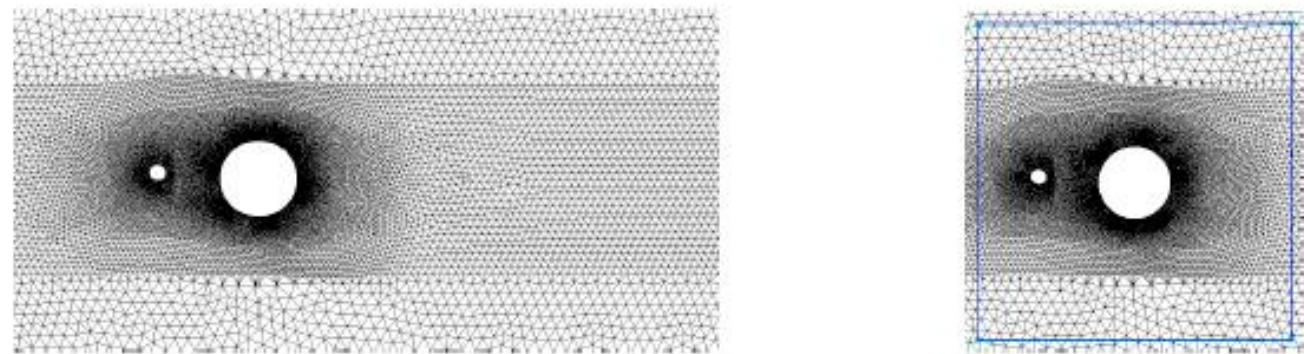

(a) Zoom around the cylinders for the modified geometry (left) and the bounding box for mesh morphing (right)

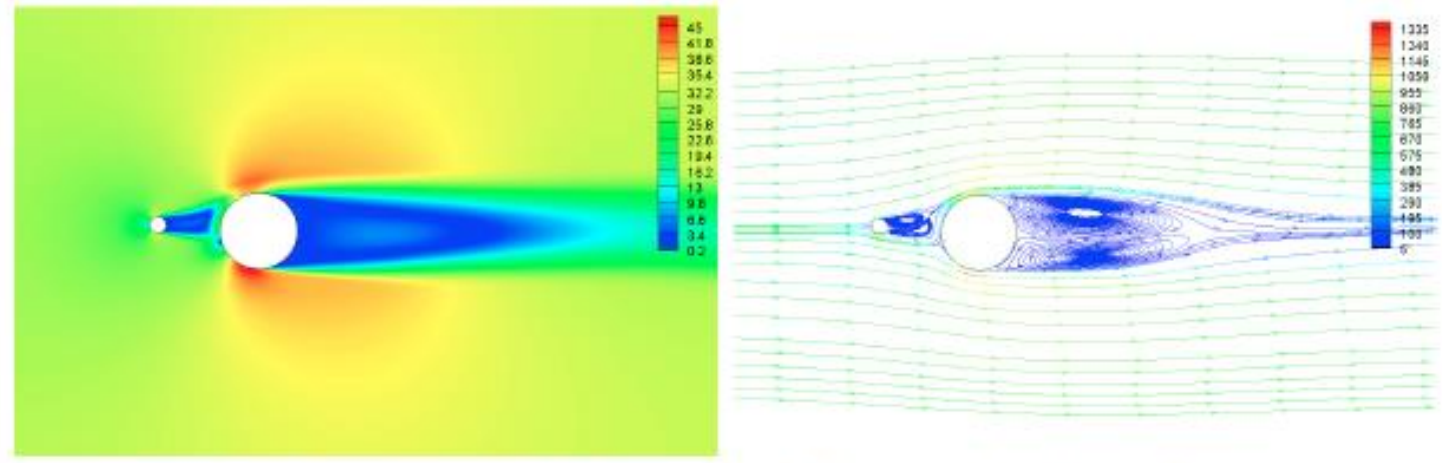

(b) Contour plot and stream function plot for the time-averaged velocity magnitude
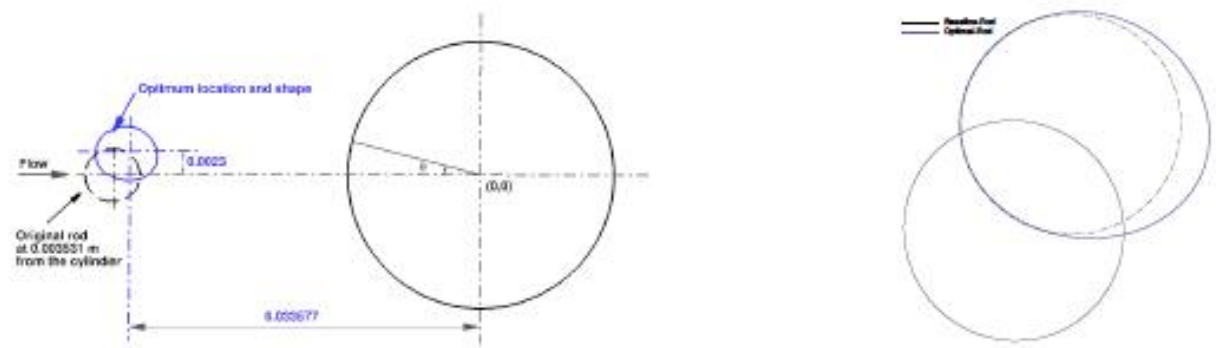

(c) Location and shape for the modified rod (left) and zoom around the rods (right).
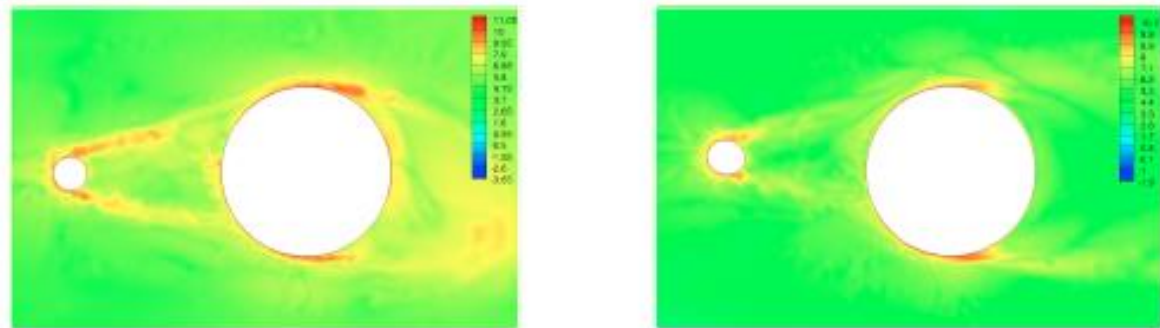

(d) Log10(Shape Sensitivity Magnitude) before modification (left) and after it (right).

Fig. 6. The flow pattern using the optimal geometry. 

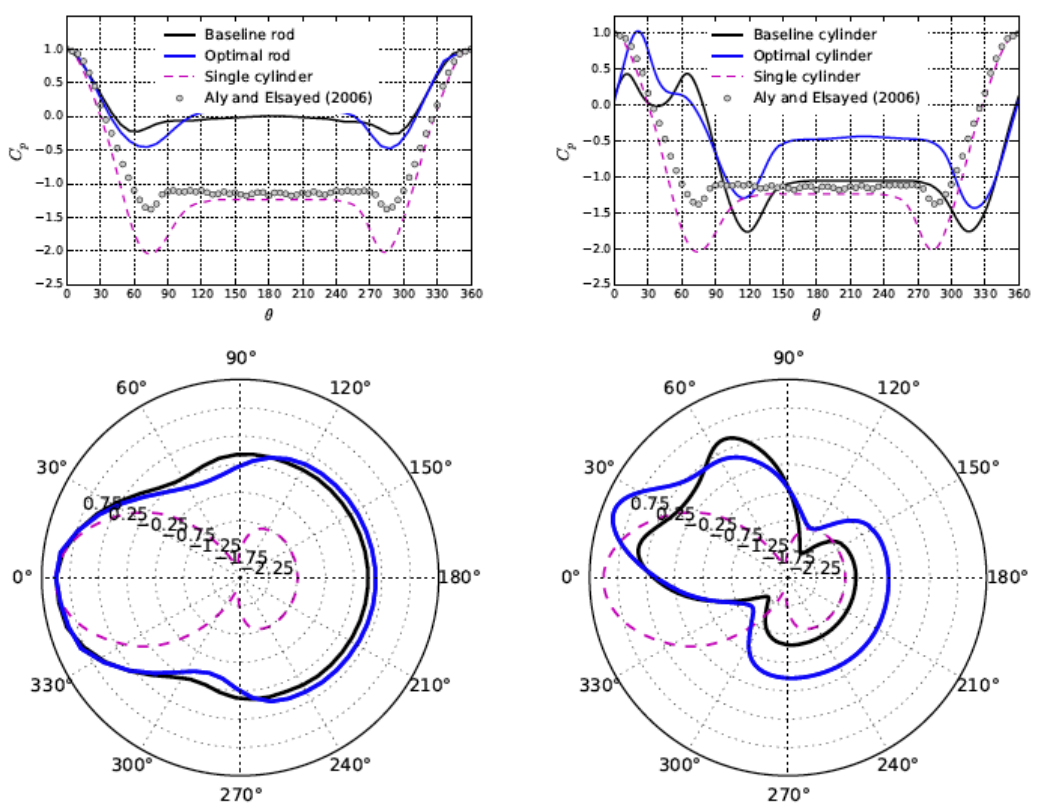

(a) The pressure coefficient
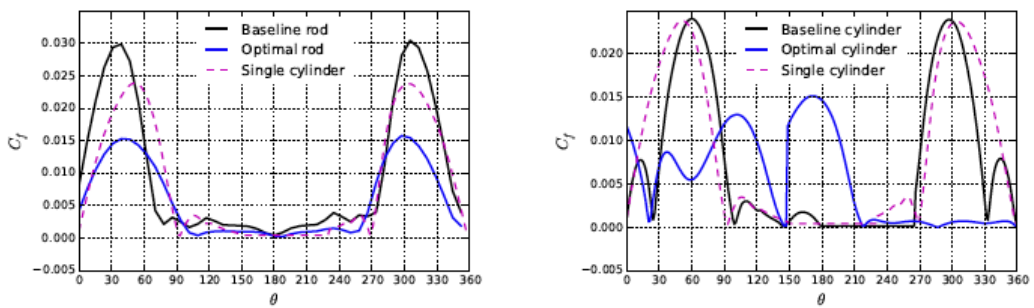

(b) The skin friction coefficien

Fig. 7. The time averaged static pressure coefficient for the upstream control rod (left) and the downstream cylinder (right).
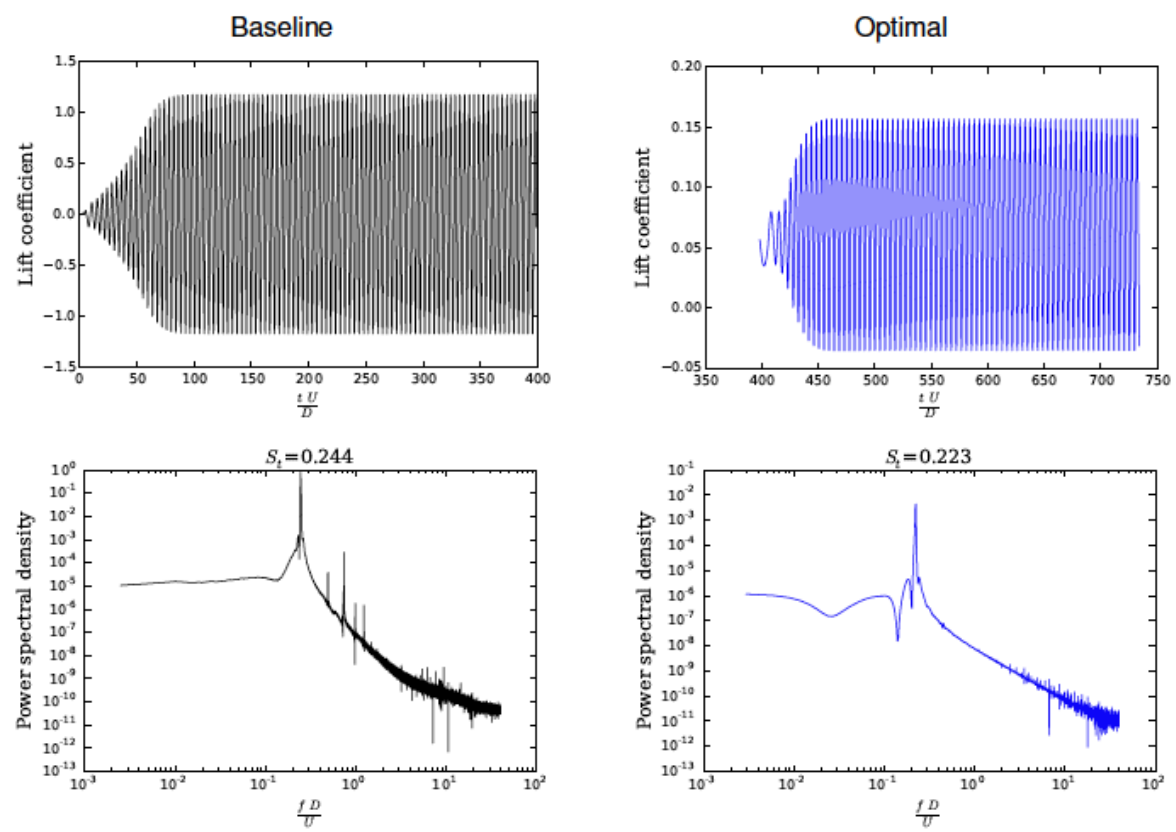

Fig. 8. The time evolution of the lift coefficient for the cylinder and the corresponding power spectral density. 